\title{
DEFECT PROFILE INDUCED BY FRICTION AND WEAR PROCESSES DETECTED BY POSITRON ANNIHILATION METHOD
}

\author{
J. Dryzek, E. DrYzeK \\ Institute of Nuclear Physics, Radzikowskiego 152, 31-342 Kraków, Poland
}

AND B. CLEFF

Institute of Nuclear Physics, University of Münster

Wilhelm-Klemm-Strasse 9, D-4400 Münster, Germany

The positron annihilation studies of defect profile in Cu samples whose surfaces were exposed to the friction and wear processes are presented. The values of the $S$-parameter and its dependences on the depth from the $\mathrm{Cu}$ surface are the functions of the value of the load applied in the sliding contact between two metals. It indicates possibilities of applying the presented measurement method in the industry.

PACS numbers: 78.70.Bj

\section{Introduction}

Contact between metallic surfaces when relative sliding motion occurs always produces plastic deformation in and around the real area of solid/solid contact. The deformation is caused by the normal and shear tractions at the contact interface and is an integral part of the friction and wear processes. The microscopic examination of the highly deformed near-surface regions has shown that plastic deformation plays an important role in sliding wear, abrasion, and erosion processes [1-3]. The deformation results in void formation, crack nucleation and propagation, extrusion and other processes that lead to the material removal. The microstructural parameters of particular interest which influence deformation and fracture include the crystal structure type, degree of chemical order, the presence of grain boundaries, stacking faults (first of all different types of dislocations), second phases and precipitates. All those processes are extensively investigated using optical microscopy, scanning and transmission electron microscopy, $\mathrm{X}$-ray and electron diffraction, Auger and X-ray photoelectron spectroscopy and mechanical properties measurements [4]. (We have not found any reports on using positron annihilation techniques.) The report presents the preliminary studies of defect profile caused by friction and wear processes in well-annealed copper samples of technical purity. 


\section{Experimental}

The Cu samples in the form of disks ( $5 \mathrm{~mm}$ thick and $15 \mathrm{~mm}$ radius) were annealed at high temperature $\left(900^{\circ} \mathrm{C}\right)$ for three hours and then slowly cooled to room temperature. The heat treatment was performed in a vacuum heater under the pressure $5 \times 10^{-4} \mathrm{Tr}$. A well-annealed Cu sample disk free of defects and positron traps was mounted on the rotated support and the small steel (100Cr6/Martensit, hardened) ball ( $4 \mathrm{~mm}$ diameter) was forced with the load perpendicularly to the disk's surface. The Cu samples were deformed in sliding contact with steel. The samples were subjected to the continuous sliding test in the pin-on-disc tribometer [5]. The disk was rotated over $30 \mathrm{~min}$ and the ball scratched a trace on the surface with the velocity $5 \mathrm{~cm} / \mathrm{s}$. Five traces were made on the disk's surface in this way. After then, the disk was cut into two symmetrical pieces in order to get two identical samples for measurements of the classical line shape $S$-parameter using the Doppler broadening annihilation line spectrometer with $1.63 \mathrm{keV} \mathrm{FWHM}$ resolution interpolated at $511 \mathrm{keV}$. (The Doppler broadening $S$-parameter [6] is defined as the ratio of the area under the fixed central channels to the total area under the annihilation line.) The small ${ }^{22} \mathrm{Na}$ source of size $4 \mathrm{~mm} \times 4 \mathrm{~mm}$, which covered the damaged area of the sample was put on the treated surface and the $S$-parameter was measured. Finally, the two pieces of the disk were etched in nitric acid in order to remove the layer $c a .20-30 \mu \mathrm{m}$ thick from the sample's surface. The $S$-parameter was measured once more with a piece of the damage surface layer removed. In such a way several layers were removed and the appropriate values of the $S$-parameters were measured. Therefore, the $S$-parameter as a function of defect profile was obtained. The defects appeared during the wear of the $\mathrm{Cu}$ disk scratched by the steel ball.

\section{Results and discussion}

Figures $1 \mathrm{a}, \mathrm{b}$ and $\mathrm{c}$ present the results of the measurement for three loads $250 \mathrm{~g}, 114 \mathrm{~g}$ and $50 \mathrm{~g}$, respectively. The thickness of the removed layer or depth from the initial disk's surface is shown on the $x$-axis. From the figures one can see that the $S$-parameter exhibits a characteristic dependence for all three loads. For all cases, the $S$-parameter decreases when the number of etched layers increases and it reaches the constant value which is equal to the value measured for the annealed defect-free sample (the bulk value). The initial value of the $S$-parameter measured on the damaged surface before etching is higher for higher load applied. For higher load, the final $z$-depth for which the bulk value of the $S$-parameter is reached also increases. All that indicates that the detected dependencies of the $S$-parameter on the $z$-depth reflect the distribution of defects produced during the wear and friction processes.

It is interesting to compare the results obtained with description of plastic deformation region induced by the ball on the disk. Figure 2 presents the diagram of full plastic flow with the onset of plastic flow for the ball contact model. That idealized schema shows the stage of the plastic deformation of the flat surface with the characteristic radius of a circular region given by the following formula [7]:

$$
a=\sqrt[3]{\frac{3}{4} \frac{M g}{E} R},
$$



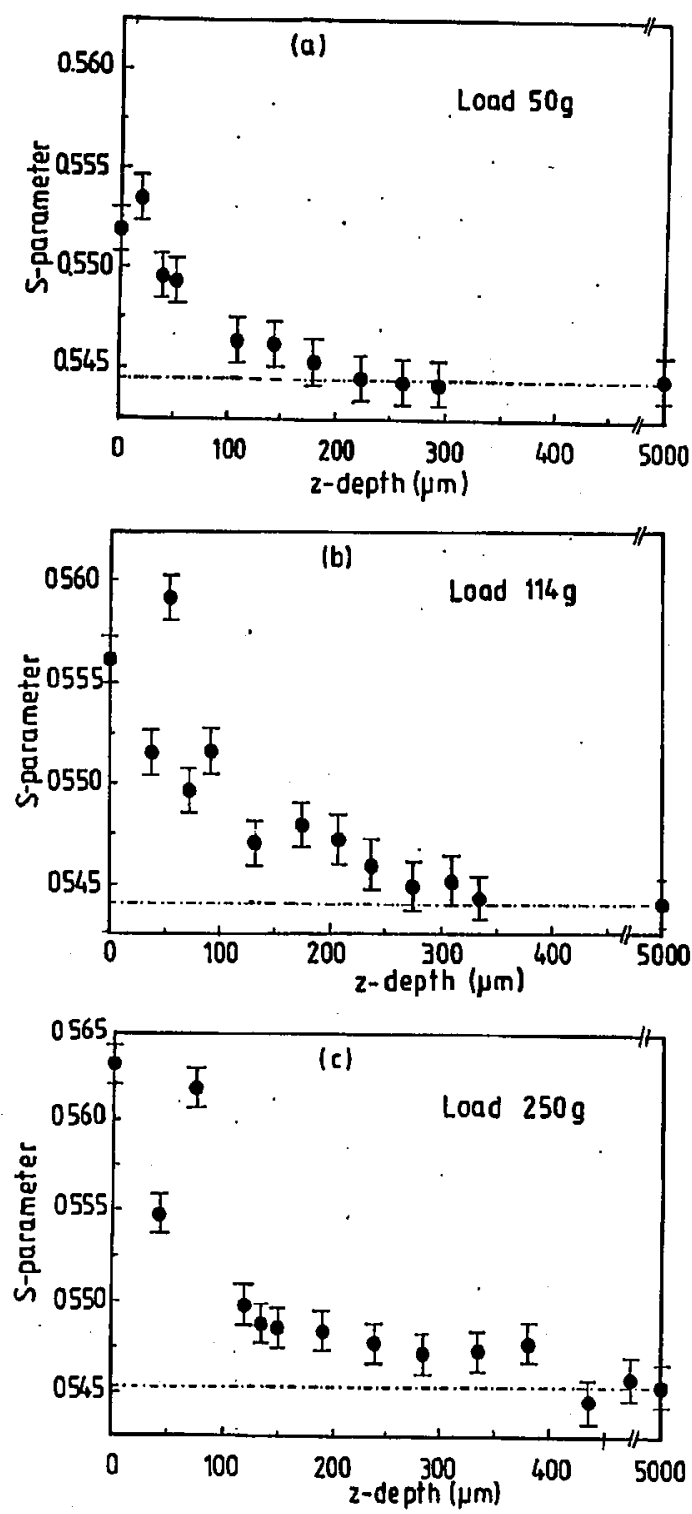

Fig. 1. The $S$-parameter as a function of the depth $z$ from the Cu disk's surface for the load $50 \mathrm{~g}$ (a), $114 \mathrm{~g}$ (b) and $250 \mathrm{~g}$ (c). The solid line corresponds to the schematic draw of positron implantation range.

where $R$ is the radius of the ball, $E$ is the elastic (Young's) modulus of the disk, $M$ is the mass of the load and $g$ is the acceleration of gravity. 




Fig. 2. The schematic diagram showing onset of plastic flow of the soft flat under a hard spherical surface.

The contact area $\left(\pi a^{2}\right)$ due to the maximum plastic deformation of the ball and the disk increased plastically by sinking of the ball into the flat. Taking into account the case of $\mathrm{Cu}$ disk $\left(E=8 \times 10^{10} \mathrm{~N} / \mathrm{m}^{2}\right)$ one can get for the highest load $a=35 \mu \mathrm{m}$ which determines the onset and the range of the plastic flow. But according to the $S$-parameter measurements this active range is much larger (extends up to $400 \mu \mathrm{m}$ ). It indicates that production of defects (vacancies and dislocations during friction processes) penetrates much deeper in bulk material than it is predicted by traditional mechanical approach. Further studies of positron lifetime spectra could give more details about type of defects located in the layer.

\section{Conclusions}

Summing up, the preliminary measurements shows that the positron technique used is sensitive enough to the defect depth profile appearing in the sliding contact between two sliding metals. The figures contain also some details of the defect profile, whose interpretation is not presented here. Our further studies will be performed in order to observe effect of other parameters e.g. temperature, velocity on the production of defects in the sliding contact of two metals and to refer to other theories of wear and friction especially those which consider the role of dislocations in microscopic processes.

\section{Acknowledgments}

The authors would like to thank Stiftung für Deutsch-Polnische Zusammenarbeit for supporting this work under the project No. 538/92. 


\section{References}

[1] N.P. Suh, Wear 44, 1 (1977).

[2] M.A. Moore, R.M. Douthwaite, Metall. Trans. A 7, 1833 (1976).

[3] S. Jahanmir, Wear 61, 309 (1980).

[4] J.J. Wert, Key Eng. Mater. 33, 101 (1989).

[5] ASLE Calalog of Friction and Wear Devices, American Society of Lubrication Engineers, Park Ridge 1976.

[6] R.N. West, in: Positron in Solids, Ed. P. Hautojärvi, Springer Verlag, Berlin 1979, p. 89.

[7] T.F.J. Quinn, Physical Analysis for Tribology, Cambridge University Press, Cambridge 1983, p. 10. 